\title{
About the influence of wheel slide protection devices action on longitudinal dynamic of trains
}

\author{
Cătălin Cruceanu*, Mădălina Dumitriu, and Camil Crăciun \\ University Politehnica of Bucharest, Department of Railway Vehicles, 313 Splaiul Independenţei, \\ 060042, Bucharest, Romania
}

\begin{abstract}
Wheel slide protection devices (WSPD) are destined to avoid important sliding of wheels during braking actions in case of temporarily impaired wheel-rail adhesion by correspondent reductions of air pressure in brake cylinders. Accordingly, longer braking distances and higher longitudinal in-train forces occur, potentially affecting the traffic safety and the passengers comfort. A general analyse and evaluation of these effects are the main targets of the present study. The theoretical considerations are sustained by simulations of braking process, considering situations of normal, respectively diminished wheel-rail adhesion determining random actuations of WSPD. The air pressure evolution in brake cylinders of was experimentally determined on a computerized brake system test stand and adequately used as input in simulations. The results indicate an increase of in-train forces and a more complex evolution of the longitudinal dynamic actions between the vehicles in the case of degraded wheel-rail adhesion, when the random actuation of WSPD have major influence in the whole braking process.
\end{abstract}

\section{Introduction}

Train braking is a complex process, influenced by numerous operational, technical and constructive factors. Specific aspects contribute to generate in-train forces that, in certain situations, may affect the train integrity and safety. Hence, it is to notice a growing interest on longitudinal dynamics of trains with the aim to define appropriate recommendations regarding both operating and design of railway vehicles, in order to recommend improvements of safety and energy efficiency of this transport mode, e.g. [1-5]. Most of the studies are focused on long heavy freight trains featured with brake blocks and UIC brake system, operating in slow-action mode. In comparison, passenger trains are shorter, lighter and more uniform in consist. Destined to run with higher speeds, the braking system require more sophisticated equipment, determining particular braking characteristics that consequently have major influences on the longitudinal dynamics of trains.

For railway transport, the deceleration ability is limited by the adhesion between wheel and rail. As an open system, the wheel-rail contact is exposed to environmental factors and contaminants that randomly may result in lowering in a very large extent the adhesion on the railhead. Braking actions on slippery track increase the braking distance and the blockage or

${ }^{*}$ Corresponding author: c_cruceanu@yahoo.com 
partial blockage of wheelset conducts to wheel flats, affecting the safety of the traffic, the vehicle, the track and the passengers comfort [6-7].

In order to provide high braking power to satisfy the safety and operational requests, for passenger vehicles, systems are usually designed at the limits of wheel-rail adhesion. This results in a pronounced sensitivity of the braking system to degraded adhesion [8]. Consequently, all passenger vehicles are featured with WSPD that, in case of important sliding, rapidly diminish the air pressure in brake cylinders until the affected wheelset returns to normal rotational speed according to the translational speed of the vehicle.

In such situations, important random variations of the vehicles' braking forces affect the whole longitudinal dynamics of the train. Supplementary longitudinal oscillations are generated in the train body, while the magnitude, time evolution and disposition of compressive and tensile forces between adjoined vehicles are completely modified.

The main aim of the study is to evaluate in certain particular situations the effects of WPSD during braking in low adhesion conditions on the in-train forces. The approach towards the subject is by simulations, using an original program in which measured air pressure variations in brake cylinder while actuating a mechanical WPSD are used as input.

While, to the authors' knowledge, no such studies have been published before, the investigations are focused on a very simple composition of the train and the simulation program involves certain assumptions to avoid, as much as possible, the interference of other parameters in the results obtained.

\section{Theoretical aspects}

Mechanical and pneumatic modelling of trains has been discussed in an extensive of publications, see for instance [1-5, 8-12].

In the present work, the train is classically considered an elastic-damped lumped system of $n$ individual rigid masses $m_{i}$ representing each vehicle, connected by couplings denoting well defined elastic and damping equivalent characteristics (Fig. 1).

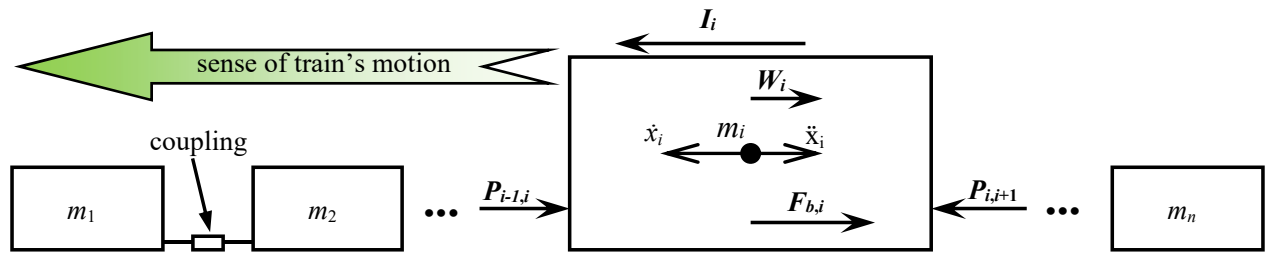

Fig. 1. Mechanical model of the train.

Assuming a track with no curves or slopes and considering the multibody formulation, the displacement $x_{i}$ of the generalized " $i$ "-th vehicle of the train is governed by the following forces: the braking forces $F_{b, i}$ the resistances $W_{i}$, the in-train forces $P_{i-1, i}$ and $P_{i, i+1}$ acting in the connections with neighboured vehicles and the inertial forces $I_{i}$ (see Fig. 1). The equation of motion is:

$$
m_{i} \cdot \ddot{x}_{i}+P_{i, i+1}-F_{b, i}-W_{i}-P_{i-1, i}=0
$$

Applied to the vehicles of the train, considering $i \in[1, n]$ and attaching the boundary conditions $P_{0,1}=P_{n, n+1}=0$, eq. (1) defines the differential equation system of second order describing the movements of the system presented in Fig. 1.

In the present study the forces in couplers were evaluated assuming an algebraic sum of elastic and friction forces, considering RINGFEDER-type buffers built in Romania by ICPVA-SA featuring trailed passenger vehicles. There were determined [11]: 


$$
P_{i, j}\left(\Delta x_{i, j}, \Delta \dot{x}_{i, j}\right)=\left\{\begin{array}{c}
k_{b} \cdot \Delta x_{i, j}+c_{b} \cdot\left|\Delta x_{i, j}\right| \cdot \tanh \left(u \cdot \Delta \dot{x}_{i, j}\right), \text { for } \Delta x_{i, j}<0 ; \\
0, \text { if } \Delta x_{i, j}<0 ; \\
k_{t} \cdot \Delta x_{i, j}+c_{t} \cdot\left|\Delta x_{i, j}\right| \cdot \tanh \left(u \cdot \Delta \dot{x}_{i, j}\right), \text { for } \Delta x_{i, j}>0
\end{array}\right.
$$

In eq. (2) $k$ and $c$ are equivalent constants depending on the elastic, respectively friction characteristics between the metallic rings inside, indexes " $b$ " and " $t$ " are referring to buffer and traction devices, $\Delta x_{i, j}$ is the stroke, $\Delta \dot{x}_{i, j}$ the relative speed for the considered element of the couplers between $i$ and $j$ adjacent vehicles of the train, $u>>1$ is a scaling factor.

Considering the vehicles designed for a specific running speed $V_{\max } \geq 160 \mathrm{~km} / \mathrm{h}$ equipped with disc brakes, the brake force is described accounting the dependency on instantaneous air pressure in brake cylinders $p_{B C}$ and the wheel-rail adhesion coefficient in normal conditions $[8,13]$ :

$$
F_{b, i}(t)=\left\{\begin{array}{l}
0, \text { if } p_{B C, i}(t)<0.4 \text { bar, else } \\
\frac{p_{B C, i}(t)}{p_{B C, \max }} \cdot m_{i} \cdot g \cdot \frac{0.33}{1+0.011 \cdot V_{\max }}
\end{array}\right.
$$

In eq. (3), $p_{B C, \max }$ is the maximum pressure in brake cylinders for emergency braking and $g=9.81 \mathrm{~m} / \mathrm{s}^{2}$ is the gravitational acceleration.

Regarding the pneumatic processes, the main aspects involved were approached as follows: an average transmission speed for brake actuation of $250 \mathrm{~m} / \mathrm{s}$ in long of the train and experimentally determined data, adequately implemented into the simulation program, for the air pressure evolution in the brake cylinders.

The operating principle of WSPD is illustrated in Fig. 2. Once the braking force become greater than the wheel-rail adhesion force (1), important sliding occurs. The system experience differences between the translational speed of vehicle and the peripheral speed of wheels. When a certain threshold value is exceeded $\left(l^{\prime}\right)$, the air from the brake cylinders is rapidly evacuated ( 1 '), resulting a decrease in braking force. When becomes lower than the actual exploitable adhesion (2), the wheelset accelerates to return to normal rotational speed (2'). This process engenders for the air pressure in brake cylinders (2') a prompt return to the initially commanded level, unless no other overbraking tendency.

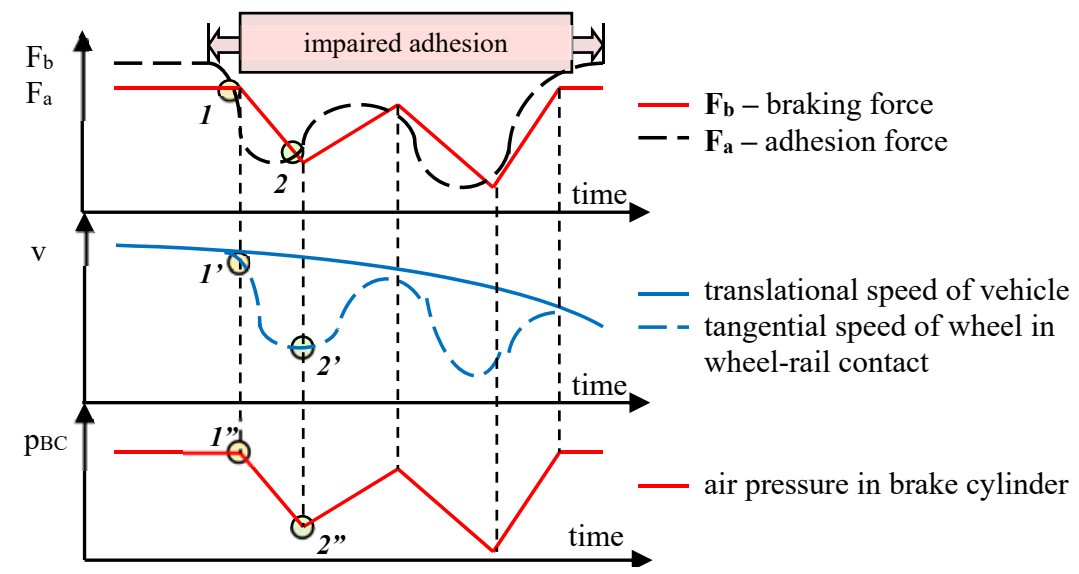

Fig. 2. Operating principle of WSPD.

The entire procedure is repeated until the commanded braking force is steadily lower than wheel-rail adhesion force, i.e. until the slippery track section is travelled by the vehicle. As 
parts of a train, the vehicles reach successively such areas and the random specific operation of each WSPD conduct to important instantaneous differences in braking forces. These definitely influence the longitudinal dynamics of the whole train.

\section{Input data and assumptions}

In order to get an image on the effects of WSPD actions on in-train forces in case of low wheel-rail adhesion, several simulations were performed. As a primary stage in our studies, certain constraints and simplifying hypotheses were assumed. The most relevant are outlined as follows: short passenger train of four identical vehicles equipped with disc brakes, KE 1 air distributor and mechanical WSPD type M2; vehicles have classical couplers consisting of RINGFEDER type buffers and traction devices; screw couplings tightened up, with no clearance between the buffer discs; the train is submitted to an emergency braking performed at $160 \mathrm{~km} / \mathrm{h}$ on a straight track with no slopes; the vehicles reach successively the low adhesion area, the moment of WSPD actuation depending on the running speed and position of each vehicle in the train composition; identical filling characteristics of brake cylinders; identical response of vehicles' WSPD after actuation.

The main input data are presented in table 1 .

Table 1. Main input data.

\begin{tabular}{|l|c|c|}
\hline Vehicle weight [t] & \multicolumn{2}{|c|}{50} \\
\hline Vehicle length [m] & \multicolumn{2}{|c|}{25} \\
\hline \multirow{2}{*}{ Specific constants for couplings in eq. (2) [N/m] } & $k_{b}=2.8 \cdot 10^{6}$ & $c_{b}=1.4 \cdot 10^{6}$ \\
\cline { 2 - 3 } & $k_{t}=5.46 \cdot 10^{6}$ & $c_{t}=2.43 \cdot 10^{6}$ \\
\hline Maximum brake force/vehicle [kN] & \multicolumn{2}{|c|}{58.65} \\
\hline Maximum air pressure in brake cylinders [bar] & \multicolumn{2}{|c|}{3.837} \\
\hline Filling time of brake cylinders [s] & \multicolumn{2}{|c|}{3.4} \\
\hline
\end{tabular}

The air pressure evolutions in brake cylinders for emergency braking, with and without WSPD actuation, were experimentally determined on the computerised system in our Brakes Laboratory. Detailed information about it and equipment can be found in [14]. The simulation program [10-11] was improved by adequately implementation of the acquisitioned pressure data. It was considered a length of about $205 \mathrm{~m}$ for the impaired adhesion section, after reaching the maximum pressure in brake cylinders (Fig. 3).

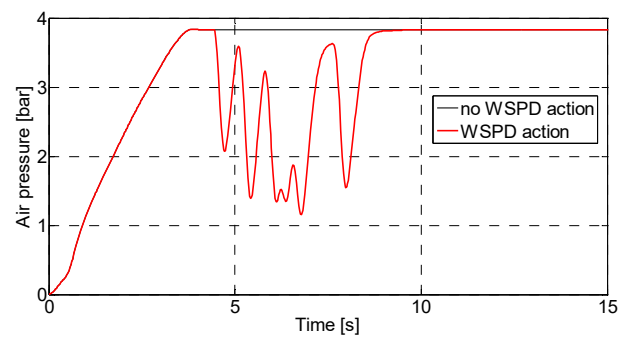

Fig. 3. Experimentally determined air pressure evolutions in brake cylinders, emergency braking.

For the sake of illustration, it is to notice that the stopping distances resulted (following [13]) by simulations for individual vehicle are $915 \mathrm{~m}$, respectively $988 \mathrm{~m}$.

\section{Results, comments and conclusions}

The main outputs achieved by simulations are the maximum values of buff and draft forces in couplers (see table 2) and the diagrams presenting time evolution of in-train forces. In Fig. 
4 are presented some of the most representative: case 0000 (no WSPD action) - considered as baseline, cases 1101 and 0111 (maximum tensile, respectively compression forces between adjacent vehicles) and case 1111 (all vehicles affected by low adhesion).

Table 2. Main input data.

\begin{tabular}{|c|c|c|c|c|c|c|}
\hline & \multicolumn{3}{|c|}{ Maximum buff forces $[\mathrm{kN}]$} & \multicolumn{3}{c|}{ Maximum draft forces [kN] } \\
\hline case*/coupler & $1-2$ & $2-3$ & $3-4$ & $1-1$ & $2-3$ & $3-4$ \\
\hline 0000 & 8.2 & 13.0 & 15.3 & -2.0 & -3.7 & -4.1 \\
\hline 1000 & 14.1 & 13.0 & 15.5 & -39.1 & -38.9 & -35.2 \\
\hline 0100 & 19.1 & 13.0 & 15.3 & -7.4 & -26.5 & -23.5 \\
\hline 0010 & 18.0 & 23.2 & 15.3 & -10.4 & -12.1 & -20.1 \\
\hline 0001 & 29.0 & 36.0 & 32.1 & -14.3 & -26.8 & -24.8 \\
\hline 1100 & 23.6 & 13.0 & 15.3 & -50.0 & -45.1 & -23.9 \\
\hline 1010 & 25.4 & 35.2 & 15.3 & -46.4 & -40.5 & -37.2 \\
\hline 1001 & 32.7 & 44.0 & 44.4 & -44.6 & -36.8 & -29.3 \\
\hline 0110 & 35.2 & 34.7 & 15.3 & -16.7 & -36.0 & -33.2 \\
\hline 0101 & 37.5 & 36.5 & 38.8 & -20.8 & -29.4 & -30.6 \\
\hline 0011 & 24.2 & 49.1 & 38.8 & -17.5 & -17.9 & -39.1 \\
\hline 1110 & 27.6 & 19.8 & 15.3 & -57.9 & -54.9 & -27.3 \\
\hline $\mathbf{1 1 0 1}$ & 33.4 & 37.9 & 38.3 & $-\mathbf{5 9 . 7}$ & -48.8 & -38.6 \\
\hline 1011 & 42.6 & 51.9 & 31.9 & -45.1 & -40.5 & -33.6 \\
\hline $\mathbf{0 1 1 1}$ & 52.1 & $\mathbf{5 2 . 6}$ & 39.7 & -22.3 & -32.6 & -22.5 \\
\hline 1111 & 48.7 & 47.4 & 37.1 & -57.9 & -53.7 & -29.6 \\
\hline$* 0-$ vehicle & with no WSPD action during braking; $1-$ vehicle with WSPD actuation \\
\hline
\end{tabular}
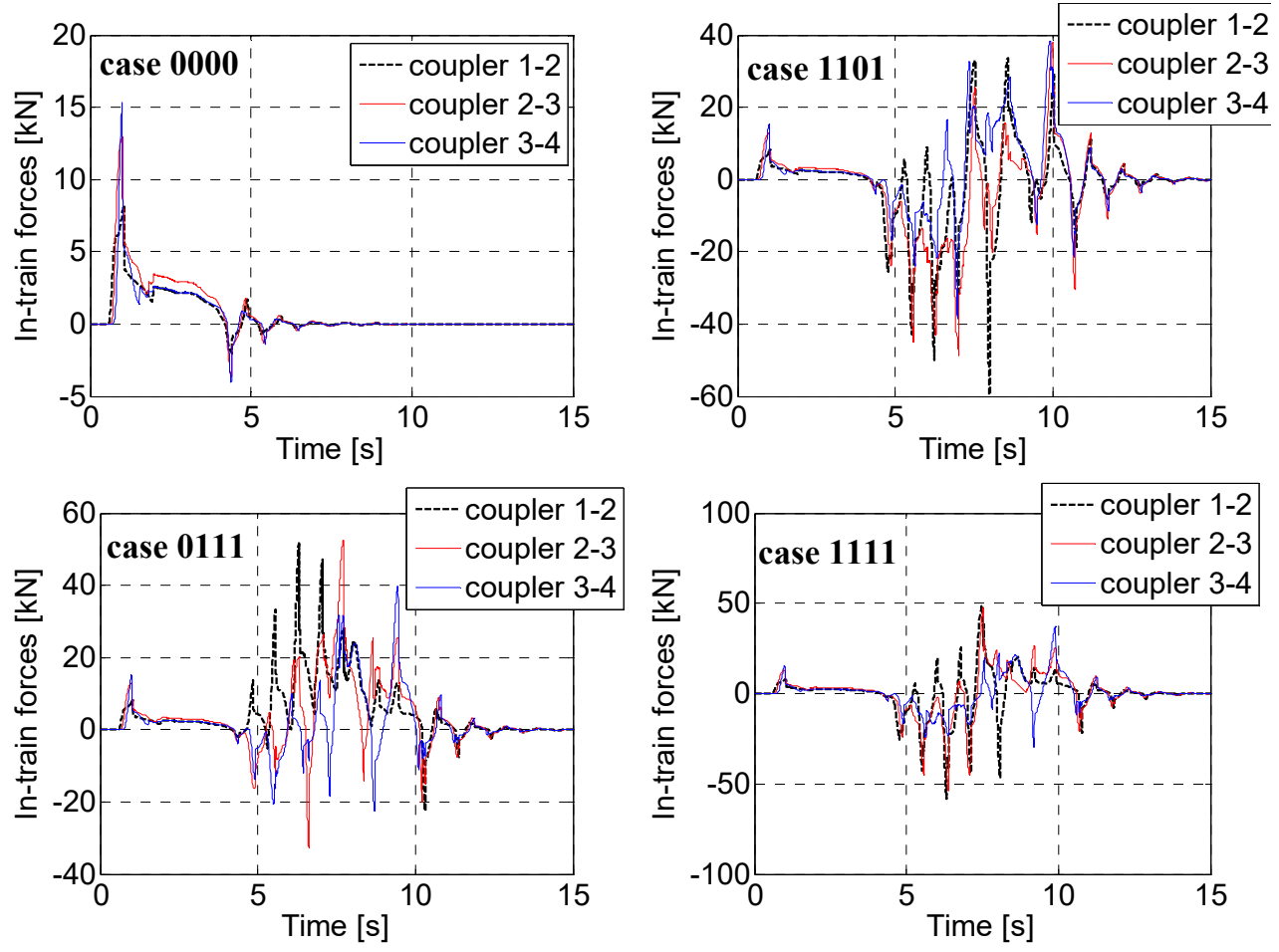

Fig. 4. Time history of in-train forces evolution (buff forces positives, draft forces negatives). 
It can be seen that during the first $4.44 \mathrm{~s}$ (until the train reaches the low adhesion sector) the braking process unfolds identically in all cases. Beyond this point, the diagrams analyse highlight both substantial modification of the whole longitudinal dynamics of the train and important increase of in-train forces. In the presented case study, considering the particular situations and assumptions presented in section 3, whenever WSPD actions are involved, the magnitude of buff and draft forces increase. These growths can exceed in certain cases more than 3 , respectively 14 times the dynamic longitudinal forces between adjoined vehicles in train resulted in the baseline case (good wheel-rail adhesion, no WSPD actuations). Even so, these forces are low enough as not to endanger the traffic safety.

It is also interesting to notice that the highest levels of in-train forces do not correspond to the case of actuation for all WSPD, but on only three vehicles of the train.

One still may note that for simulations was selected a mechanical type of WSPD, known to operate simultaneous on all wheelsets of the vehicle and to engender high variations of pressure in brake cylinders. In case of featuring the vehicles with electronic WSPD, lower effects are expected on in-train forces in the same adhesion conditions.

In conclusion, WSPD are necessary for the traffic safety and mandatory for passenger and traction rail vehicles, requirement imposed by international regulations. Our study highlights certain complementary aspects on the complexity of the train braking process. The actuations of WSPD during braking actions performed in low adhesion conditions engender longer stopping distances, considerable modifications in longitudinal dynamics of the train and significant increase of in-train forces. Obviously, for a more complete picture of effects, more work is required but, at the moment, our approach represents at least a starting point and a source of interesting aspects in passenger trains braking studies.

This work has been funded by University Politehnica of Bucharest, through the "Excellence Research Grants" Program, UPB - GEX - 2016. Research project title: Research on developing mechanical and numerical models for the virtual evaluation of the dynamic performances in the railway vehicles (in Romanian). Contract number: 48/26.09.2016.

\section{References}

1. C. Cole, Longitudinal train dynamics, in Handbook of railway vehicle dynamics, S. Iwnicki (Ed), Taylor \& Francis, 239-278 (2006)

2. Q. Wu, S. Luo, C. Cole, J. Mod. Transport. 22, 3, 127-136 (2014)

3. L. Pugi, D. Fioravanti, A. Rindi, 12th IFToMM World Congress, (2007)

4. A. Nasr, S. Mohammaddi, Proc. IMechE, 224, Part F: JRRT, 523-534 (2010)

5. M.J. Arsahd, Attic-ur-Rehman, A.I. Durrani, H. Ahamad, JFET, 20, 1, 38-53 (2013)

6. O. Arias-Cuevas, Low adhesion in the wheel-rail contact, (Doctoral Thesis, Delft, 2010)

7. Y. Zhu, Adhesion in the wheel-rail contact, (Doctoral Thesis, Stockholm, 2013)

8. Q. Wu, C. Cole, S. Luo, M. Spiryagina, Veh Syst Dyn 52, 6, 733-758 (2014)

9. C. Cruceanu, Train braking, in Reliability and Safety in Railway, X. Perpinya (Ed), InTech, 29-74 (2012)

10. C. Cruceanu, R. Oprea et al., Proc. of Int. Conf. on Computer Science WORLDCOMP'09, 58-64 (2009)

11. C. Crăciun, T. Mazilu, Ann. Fac. Eng. Hunedoara - Int. J. Eng., 12, 3, 19-26, (2014)

12. C. Cruceanu, C. Crăciun, AMM, 378, Trans Tech Publications, 74-81 (2013)

13. C. Cruceanu, C. I. Crăciun, I. C. Cruceanu, AMM, 809-810, 1085-1090 (2015)

14. C. Crăciun, C. Cruceanu, Brakes for railway vehicles - Applications (in Romanian), Matrixrom (2011) 